\title{
An HMM-based Multi-sensor Approach for Continuous Mobile Authentication*
}

\author{
Aditi Roy, Tzipora Halevi and Nasir Memon \\ New York University, Polytechnic School of Engineering, Brooklyn, NY, USA \\ ar3824@nyu.edu, thalevi@nyu.edu, memon@nyu.edu
}

\begin{abstract}
With the increased popularity of smart phones, there is a greater need to have a robust authentication mechanism that handles various security threats and privacy leakages effectively. This paper studies continuous authentication for touch interface based mobile devices. A Hidden Markov Model (HMM) based behavioral template training approach is presented, which does not require training data from other subjects other than the owner of the mobile device and can get updated with new data over time. The gesture patterns of the user are modeled from multiple sensors - touch, accelerometer and gyroscope data using a continuous left-right HMM. The approach models the tap and stroke patterns of a user since these are the basic and most frequently used interactions on a mobile device. To evaluate the effectiveness of the proposed method a new data set has been created from 42 users who interacted with off-the-shelf applications on their smart phones. Results show that the performance of the proposed approach is promising and potentially better than other state-of-the-art approaches.
\end{abstract}

\section{Keywords}

Touch pattern; Continuous authentication; Hidden Markov Model; Behavioral biometric; Multi-sensor

\section{INTRODUCTION}

Traditional access control mechanisms used in most smart phone systems today, like password or lock pattern, are vulnerable to various attacks, including password guessing and eavesdropping $([14,6])$.

In search of an enhanced secure authentication mechanism, various works explored graphical passwords $8,7,2,28,3,9$. Due to the growing popularity of touch-interfaces, research started exploring authentication methods that exploit touch patterns 21, 26]. Sae-Bae et. al showed that users can be authenticated from their multi-touch interaction through extraction of touch characteristics 22, 23. However, both graphical and multi-touch gestures have only been proposed at the login stage and therefore do not provide protection against unauthorized access after that stage.

Continuous authentication has been suggested as a method of providing persistent protection to the users 1 , 16]. Here,

${ }^{*}$ This paper was published as 19 .

Copyright (c) 2017 IEEE. Personal use of this material is permitted. However, permission to use this material for any other purposes must be obtained from the IEEE by sending a request to pubs-permissions@ieee.org instead of authenticating a user at the time of login, the system continuously monitors aspects of the user behavior biometrics, like keystroke dynamics [24], speaking pattern [29], device use patterns [15] and touch pattern 25, 10, 11. 4, 5] in order to maintain authentication after login.

Touch behavior biometrics provide a promising direction for continuously monitoring the user input on mobile devices. The existing approaches 25, 10, 11, 4 typically employed a binary classifier trained with the touch data from both the legitimate user as well as multiple other users. Reported results demonstrated that such classifiers detected the authentic user with high probability. However, in real-life, training a system with data from other users is not feasible.

This paper presents a Continuous HMM based Authentication System (CHAS) on touch devices. While a basic HMM-based algorithm has been developed before in [18, that method worked only on horizontal and vertical slide data 11 captured from mobile devices in constrained situation. The method has been extended here to perform recognition on general touch-pad input gestures, such as tap, up-down or forward-backward slide. In addition, it provides a method for combining data from multiple sensors, like accelerometer and gyroscope, to achieve a higher probability of detection, without interfering with the usage model of the device. The method offers the possibility of being trained using only the authentic user's data and can be updated with new data over a period of time as needed.

The proposed CHAS framework performance is demonstrated on a newly acquired data set that allows general touch-pad input by the user in a real-world scenario. In-depth analysis of the proposed method using this data set shows improved authentication accuracy can be achieved when multi-sensor data is combined with touch information.

The key contributions of this paper are thus two-fold. First, the paper introduces CHAS, an improved method for HMMbased continuous authentication, that works based on multisensor touch information. Second, the work includes a new data set that features multi-sensor data from 42 users. Unlike the previous data set that was used to test continuous mobile authentication, ([11]) which had only limited gestures (i.e., horizontal or vertical slides) created in controlled manner, the new data set captures data in unconstrained situations. This study includes extensive evaluation of the newly proposed framework on this data set.

The rest of the paper is organized as follows. Section 2 presents the survey to examine the need for continuous au- 
thentication on mobile phones. The CHAS framework is introduced in Section 3. In Section 4 the extensive experimental results are discussed and Section 5 includes the conclusions for the work.

\section{EXPLORATORY STUDY}

Previous work was developed based on the assumption that continuous authentication would be beneficial for the users and that users are worried about the data on their phone. However, no user study that the authors are aware of has been done to support this idea. In this section we present a first attempt to examine the need for continuous authentication by means of two user surveys. The surveys show that participants are concerned about the data stored on their phone and that existing locking mechanisms are not robust enough as most of the participants observed someone else's pin in the past. Therefore, the surveys demonstrate the need for the development of alternatives to pin-based or pattern based locking mechanisms.

This work includes two surveys. The first was filled by the 47 participants at a northeastern University 20 . The second was conducted online and included 267 participants 27]. Both studies were designed to look at the mobile usage and locking patterns of the participants.

The studies showed that most participants lock their phones ( $87 \%$ of the participants in the first study and $82 \%$ of the participants in the second study). However, participants in the first study were asked if they have an application that protects their apps/pictures/media files. Only 15\% of the participants indicated that is the case. Most participants in this study were worried someone would access their data in their absence (55\%). Similarly, most participants in the second study said they had data they would like to hide $(71 \%)$.

In addition, in the second study, participants were asked if they ever observed the pin of another user. $73 \%$ of the participated did observe in the past the pin of a friend or family member, and even a higher percentage (79\%) knew the current pin of someone else, a finding that raises a concern about the safety of current locking mechanisms.

The overall results can be viewed in Table 1 . Since most participants stated they have data they deem as private on the phone, these studies reveal the need to further continue looking for alternative methods for mobile authentication.

\begin{tabular}{|c|c|c|}
\hline & Study 1 & Study 2 \\
\hline \hline No Participants & 47 & 267 \\
\hline Lock the phone & $87 \%$ & $82 \%$ \\
\hline Use Pin or Pattern Lock & $87 \%$ & $81 \%$ \\
\hline Worried about Data Privacy & $55 \%$ & $71 \%$ \\
\hline Observed someone else's pin & - & $73 \%$ \\
\hline
\end{tabular}

Table 1: Survey results - Mobile User Security. Most users are using a lock mechanism and are worried about their data privacy. Most of the participants in the second study also observed someone else's pin in the past.

\section{PROPOSED HMM BASED CONTINUOUS AUTHENTICATION SYSTEM}

In this work, we assume that a malicious attacker has gained access to a person's mobile phone. The device is either unprotected (e.g., no PIN) or the attacker somehow knows the authentication secret, for instance by shoulder surfing the owner. The attacker can then perform undesirable actions with the device violating the owner's privacy in his absence or without his knowledge. The objective of the current work is to handle such situations and make this kind of manipulation impossible by analyzing touch behavior biometric of the user. To this end we introduce a new Continuous HMM based Authentication System (CHAS) described in the next section.

\subsection{Data Sources}

Usage of multiple sensors, including audio sensor, light sensor, accelerometer, gyroscope, and magnetometer have been used to provide secure access control [12,13 in mobile phones. Current work relies on touch, accelerometer, and gyroscope data to create a behavior biometric model of the user for authentication purposes.

A touch-screen based smart phone is typically operated through touch commands such as tap, slide, pinch, and free stroke handwriting. However, pinch, a two-finger gesture to do zooming, is used less than $5 \%$ of the time used by the overall gestures 4]. Similarly, though handwriting is an alternative input method to enter characters, it is not used very often. Tap and slide are the basic and most frequently used gestures. They are utilized to perform almost all of the actions on the smart phone, such as typing using soft keyboard, unlocking screen with PIN and navigating documents. Therefore, in the current work, we develop a user behavior model based on these two popular gestures.

In practice, touch gesture consists of a series of raw data input forming a touch data sequence. For each gesture performed on the touch-screen of the mobile device, we capture six raw features - time stamp, vibration, rotation, pressure, size of touch, and position. A pre-processing step is performed to normalize the sampling rate of the data sequences, using linear interpolation to derive a re-sampled signal with a uniform rate.

\subsection{Architecture of CHAS}

To design an effective touch data based user authentication approach, two key issues need to be addressed: 1) how to model the user behavior from the touch data, 2) how to recognize users according to this model. Figure 1 outlines the framework of the proposed CHAS system. Centric to this approach is HMM based pattern recognition algorithm that identifies the legitimate user from touch data. As HMM was found to be better than the exiting approaches in modeling user touch behavior [18, while employing only the owner's touch information, it is chosen as the basic classifier. However, unlike the algorithm proposed in [18, in which a different HMM system was needed for each type of movement, one architecture is created for different types of gestures in the newly proposed CHAS framework.

The proposed CHAS framework works in two steps: training and authentication. During training, a behavioral model is created based on the touch behavior (pressure, area, duration, position, vibration and rotation) of the user. At the time of authentication, the test observations are compared with the stored behavioral model to establish the identity of 


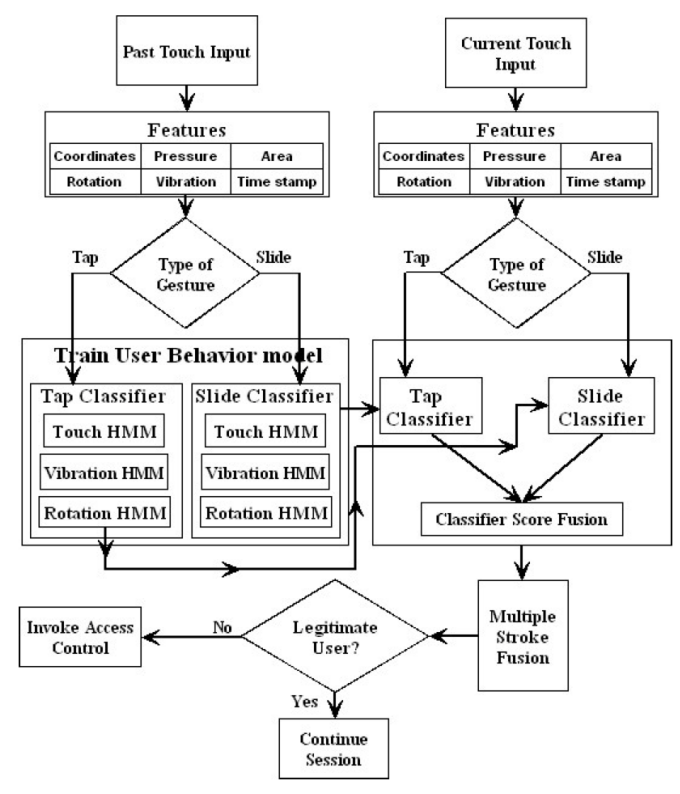

Figure 1: Overview of CHAS framework

the user.

After getting all the training touch data, the behavioral model is created based on the type of gesture. Touch-screen can capture each user touch event and generate corresponding touch data sequence. This touch data sequence is considered directly as the basic input for modeling the user behavior. However, different sequences corresponding to different types of touch gestures contain different spatio-temporal characteristics. To address this issue, the proposed approach considers each type of touch gestures separately and models them with their corresponding sequences of raw data. Since two primary gestures are considered here, i.e. tap and slide, two classifiers are developed. Unlike [18, where separate HMM models have been used to represent slides of different directions (horizontal and vertical), here one single model has been employed to represent all types of slide gestures. To achieve this, the slide gestures are normalized to make them rotation-invariant. Each classifier consists of three HMMs depending on the data source. First one is based on only touch information, i.e., coordinate, pressure, area and time stamp. The other two are corresponding to vibration and rotation data obtained from the sensors.

During deployment, given a user model and some recently observed behavior, the likelihood that the device is in the hands of the legitimate user is computed. For both the slide and tap classifier, the likelihood score obtained from the three HMMs are averaged to get a combined similarity score. This value is used as an authentication score. It may happen that the data from all the sensors is not available or usable at a certain time. Any continuous user identification approach using multiple sensors should take care of such a situation. The proposed system is flexible enough to handle subset of sensors data. It can still work efficiently and give robust performance.

To increase the robustness of the authentication method, multiple consecutive gestures are used for the final decision.
The mean of all the combined similarity scores is employed for this purpose. The score is used to make an authentication decision: typically, a threshold is used to decide whether to accept or reject the user. The threshold can vary depending on whether the application is security sensitive. If the input is detected to be of a legitimate user, he can continue using the device without any interruption. Otherwise, access control, like entering the text or pattern based password, is invoked.

\subsection{HMM based Behavior Model}

Here the basic HMM, which is used to model the touch behavior of a subject as well as other sensor data input is described. HMM is considered for modeling the touch gesture behavior of a subject since it captures the local dynamic characteristics as well as the shape and length of a gesture. The left-right topology is chosen with no state skip allowed. The observable output is the trajectory information of the gestures. The states represent the transitive properties of the consecutive coordinates of the gesture and the state sequence that maximizes the probability of observing the training gestures becomes the corresponding model of a subject.

For each gesture trajectory, an M-mixture left-to-right HMM is fitted using the Baum-Welch algorithm 17. The optimum number of states and mixtures of an HMM varies with the complexity and length of gestures in the training sequences. Five-fold cross validation principle is used to estimate the optimal number of states and the associated HMM parameters.

Once the behavioral models for all subject classes have been created through HMMs, authentication of the subjects is performed by computing the log-likelihood of the input gestures using Viterbi algorithm [17. Along with the traditional log-likelihood value, an additional measure named as gesture kinematics is computed that represents the percentage of time spent in each state. Next, the similarity scores are derived from the log-likelihood value and the gesture kinematics. These two scores are combined to get the combined similarity score for authentication. Details of the HMM based behavior model can be found in 18.

\section{EXPERIMENTAL RESULTS AND DISCUS- SION}

This section describes the performance of the proposed CHAS framework. The authentication system is evaluated through calculation of False Acceptance Rate (FAR) and False Rejection Rate (FRR). Since these two error rates are inversely related (lower FAR increases the system security while lower FRR increases its usability) Equal Error Rate (EER) is also measured, where the FAR is equal to the FRR value.

Detail evaluation has been performed to test how the CHAS framework works with different types of gestures in two possible situations: when only touch information is used and when both touch as well as sensor data are available. Experiments have been performed to test the performance of the proposed system with increasing number of consequential gestures.

\subsection{Data Set Description}

To evaluate the CHAS framework in challenging real-life scenarios, a new data set has been created that mimicked regu- 


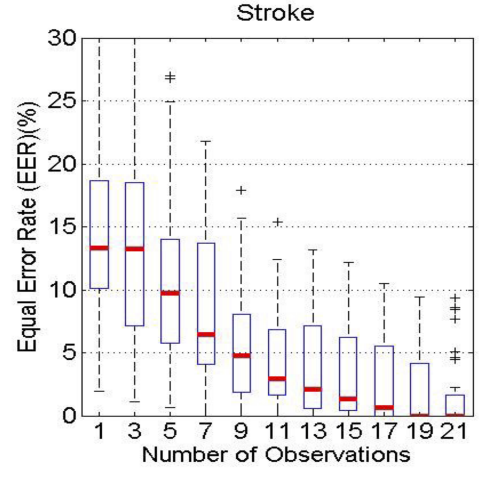

a)

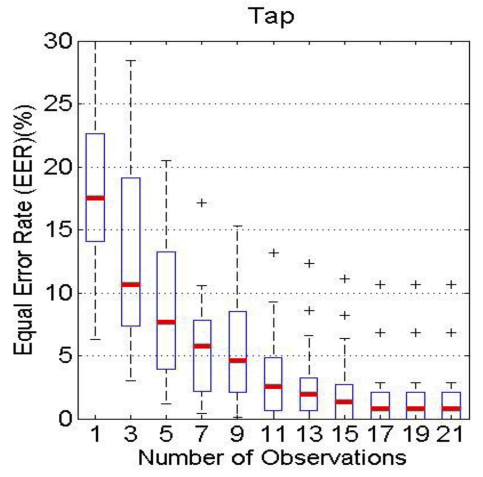

b)

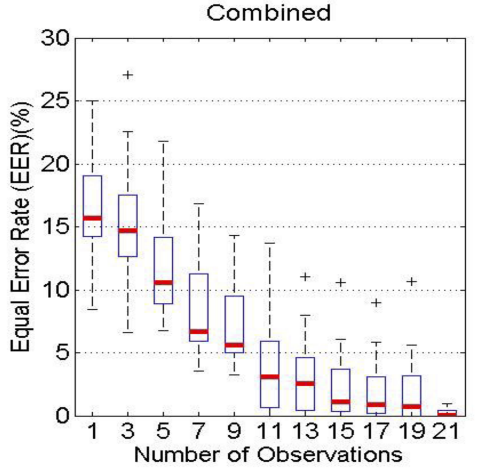

c)

Figure 2: Equal error rate variation as a function of the number of gestures using touch-pad input

lar usage of mobile devices. The users were asked to perform activities using off-the-shelf online tools, such as reading a Wikipedia page and filling Qualtrix questionnaires. This is in contrast to the data set used in 11, 18, in which a custom-application was created that enabled capturing specific horizontal and vertical movements by the user. The participants were asked to do a specific set of tasks using their smart phone. They were told the goal of the study is rating the different tasks. Those tasks included, controlling a smart phone UI using slide touch gesture (vertical and horizontal slide), mobile web browsing using pinch and spread touch gestures, entering sentences using virtual touch keyboard, selecting radio buttons by tap gesture, etc. Each task was repeated multiple times by the same user.

Data was collected from 42 users among which $75 \%$ of the participants were men, $78 \%$ of the participants belonged to the $18-24$ years age group, $15 \%$ to the 25 -30 years group and $7 \%$ to the $30-35$ years group. During data collection, users were asked to open their mailbox and read an e-mail which had detailed instructions about the sequence of tasks to be performed. Afterwards, users had to open the links which pointed to the following five tasks.

- A Wikipedia page of Niagara Falls

- Questionnaire based on Niagara Falls Wikipedia page

- A Wikipedia page of NYU Poly.

- Questionnaire based on NYU Poly Wikipedia page.

- A final survey questionnaire

Every user was asked to read through each web page and then click on a link to go to the next task. During the process, data of all types of touch events were recorded. The data includes the coordinates of touch, the pressure applied, the size of touch, and the values of the accelerometer and gyroscope sensors along with the time stamps.

The data was collected using Nexus 4 multi-touch phone with Quad-core 1.5Ghz processor, and screen resolution $768 \times$ 1280. The data capture application was developed on the Android platform 4.4.

\subsection{Authentication with Touch Data}

The performance of the CHAS framework has been evaluated from the two most frequently used gestures on mobile phone - tap and slide. In this section, we describe the authentication results using only touch information. The touch data obtained from the first two tasks described in Section 4.1 was used to train the classifiers and the data from the following three tasks was used for testing.

EER Performance: Figure 2 presents the EER performance of the CHAS framework. Since the number of consecutive gestures required to get the final classification result reflects the time requirement of the whole system, EER variation with the number of observations has been plotted here. On each box, the heavy red horizontal bar is the median, the edges of the box are the first and third quartile, the whiskers extend to the most extreme data points not considered outliers, and the outliers are plotted individually.

Figure 2(a) shows that the median EER of authentication using one observation of slide is $13.29 \%$. The EER decreases to $0 \%$ after observing 19 consecutive slide gestures. Similarly, for tap gesture, the median EER is found to be $16.55 \%$ using one observation. After 17 consecutive taps the EER saturates to near $1 \%$ (see Figure 2(b)). Since slide gesture is comparatively longer than tap, more data points are recorded for slide. The increased number of data points made the slide gesture more distinguishable than tap. Thus, the authentication performance based on slide model is better than the tap.

Next, we evaluate the performance of CHAS framework in a more general scenario where the action sequences are random combination of any types of gestures. Experimental results show that the median EER is $15.10 \%$ with one observation (see Figure 2(c)). However, after 21 observations it is reduced to $0 \%$. Thus, it may be noted that when a combination of slide and tap gestures is used, the EER becomes worse than using slide gesture alone while better than only tap gesture.

FAR and FRR Performance: We also look at the security and usability of the proposed approach in terms of FAR 


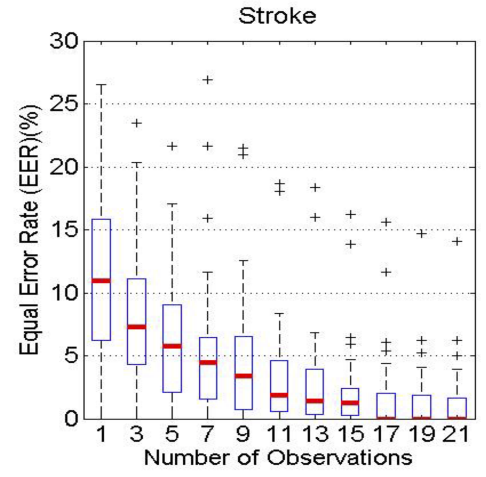

a)

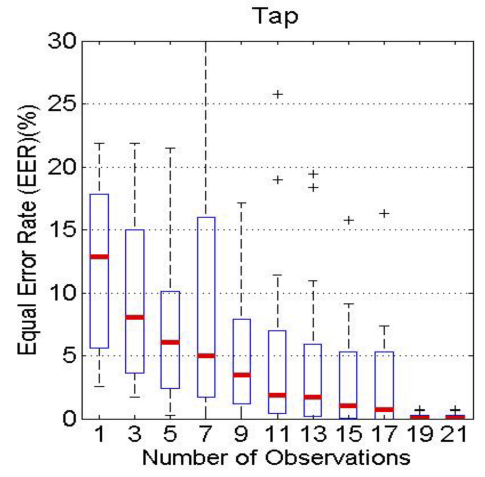

b)

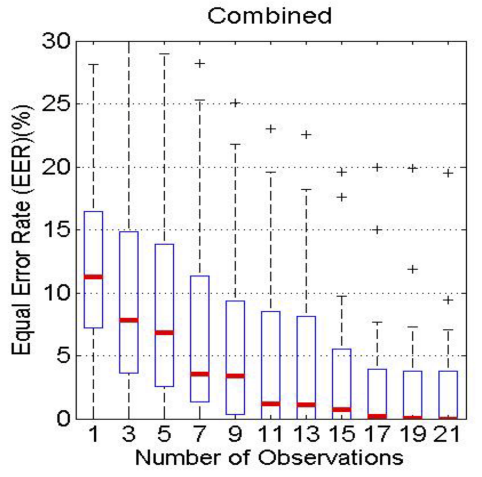

c)

Figure 3: Equal error rate variation as a function of the number of gestures using multi-sensor information

and FRR. In applications where security is not so much of importance (like games), low FRR is desired. Figure 4 depicts the median FAR performance of the system. When the FRR of the CHAS framework is zero, the median FAR of the authentication is near $0 \%$ after observing 15 taps and 17 slide gestures.

For applications with high security requirement (like banking), FRR performance of the CHAS framework is also evaluated while the FAR is set to zero (see Figure 5). It can be observed that the FRR reaches $0 \%$ after 19 consecutive taps and 17 consecutive slide gestures.

Using combined gesture data, both the FAR and FRR come down to $0 \%$ after 17 gesture observations. These results indicate inherent strength of the HMM based behavior model in varied usage scenarios.

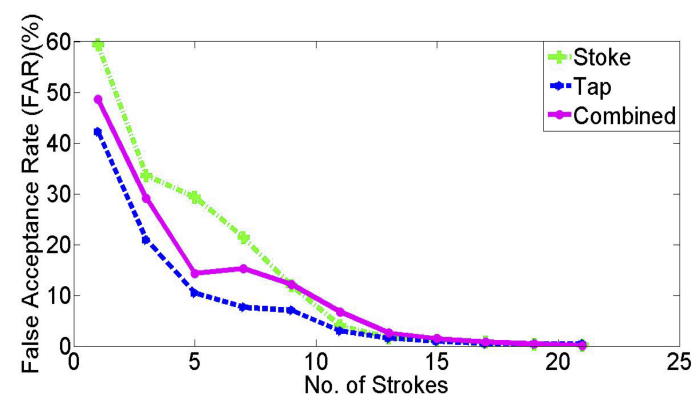

Figure 4: False acceptance rate variation as a function of the number of gestures using touch-pad input

\subsection{Authentication with Multiple Sensor Data}

In this section, we evaluate the CHAS framework using touch as well as two other sensors data. Similar to the former case, here also training is done based on the touch, accelerometer and gyroscope data obtained from the first two tasks and the rest of the data is used for testing.

EER Performance: First, we show how the system works with either tap or slide gesture (similarly to the previous

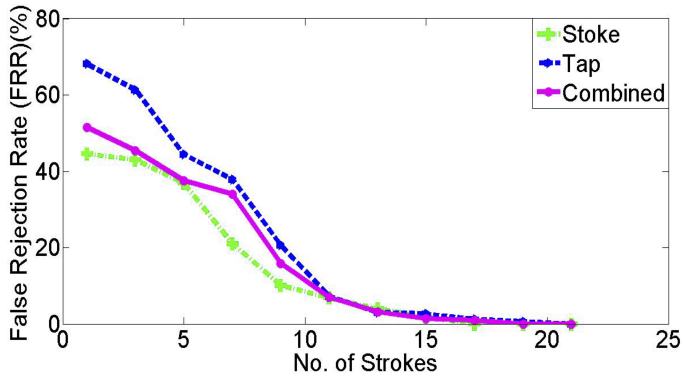

Figure 5: False rejection rate variation as a function of the number of gestures using touch-pad input

evaluation). Then, the system performance with general gesture data set that features any combination of these two gestures is reported.

Figure 3 presents the EER performance of the two gestures with increasing number of observations. It can be seen that the median EER using one observation of tap is $12.85 \%$ and one slide is $10.92 \%$. After observing about 19 consecutive taps and 17 consecutive slide gestures, the EER is reduced to $0 \%$. However, while using combined gestures, the EER is found to be $11.25 \%$. It decreases to $0 \%$ after 17 consecutive gestures. Therefore, it may be noted that inclusion of the multi-sensor data helps to improve the performance, resulting in lower EER compared to using only the touch data for the same number of consecutive gestures.

These results are comparable to the results achieved using only vertical or horizontal slide data 18,11 , where 11 consecutive strokes were needed to achieve near $0 \%$ EER. Thus, with a small increase in the number of required gestures, CHAS framework achieves similar accuracy while handling almost every possible gesture performed on a mobile device in unconstrained manner. Moreover, since this result is achieved with a combination of gestures where tap generally requires less time than slide gesture, overall time requirement to get the verification result of the CHAS framework is close to 18 . 


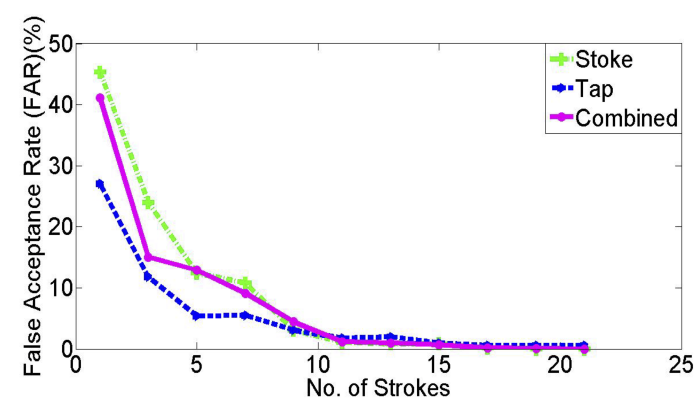

Figure 6: False acceptance rate variation as a function of the number of gestures using multi-sensor information

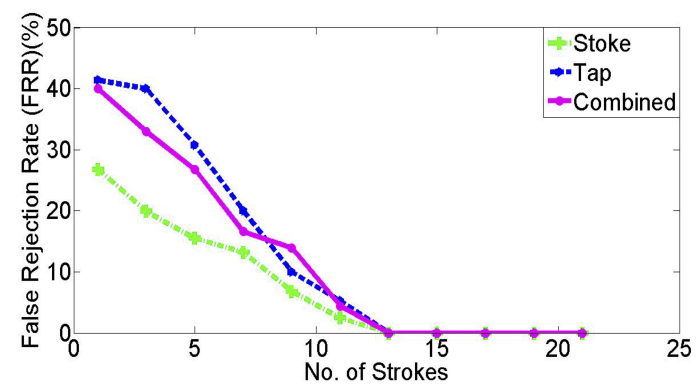

Figure 7: False rejection rate variation as a function of the number of gestures using multi-sensor information

FAR and FRR Performance: Figure 6 presents the FAR performance of the system. The results show that the median FAR reaches $0 \%$ after observing about 15 consecutive taps and 13 consecutive slide gestures. Similarly, the median FRR decreases to $0 \%$ with 13 consecutive taps or slide gestures (see Figure 7). Using combined gesture, the FAR decreases to $0 \%$ after 11 consecutive observations, while the FRR decreases to the same level with 13 gestures. Thus, the FAR and FRR performance of CHAS framework also improved after including multi-sensor data with touch information.

\section{CONCLUSIONS}

This work presents a survey that points out the need for enhanced security mechanism beyond the point of traditional login. This observation motivated us to design CHAS framework that models user touch behavior using HMM. The authentication method is based on multi-sensor data recorded from the owner's touch interactions on his mobile device. Extensive evaluation of the proposed approach on a newly acquired database, which features real-life mobile usage scenario, has been carried out.

The first part of the work demonstrates that it is possible to authenticate users with reasonable accuracy using only the touch data of the owner. The benefits of using only the device owner's data are twofold. First, in case of personal devices, data from other users may not be available. Thus, training the classifier with other users' data is not possible. Second, authentication results using only the owner's data for training reflect the real-life situation in a better way.

Next, the work continues by integrating additional data from accelerometer and gyroscope sensors. The users were not restricted to do any specific gestures on the phone but were allowed to perform different activities freely. Testing on the new acquired data set shows that the proposed framework achieves robust authentication result, with 0\% ERR using 17 consecutive observations.

The results suggest that the CHAS framework is applicable in a variety of situations, both using only touch data as well as multi-sensor data, to execute continuous authentication based on natural touch interactions. Future work involves automatic update of the CHAS framework with new data over time without the need of retraining.

\section{Acknowledgment}

This material is based in part upon work supported by the National Science Foundation under Grant No. 1228842. We would like to thank T.V. Nguyen for sharing some results of his mobile security survey with us.

\section{REFERENCES}

[1] Y. Aksari and H. Artuner. Active authentication by mouse movements. Proc. of IEEE 24th Intl' Symposium on Computer and Information Sciences (ISCIS), 2009.

[2] S. Akula and V. Devisetty. Image based registration and authentication system. Midwest Instruction and Computing Symposium, 4, 2004.

[3] J. Birget, D. Hong, and N. Memon. Graphical passwords based on robust discretization. IEEE Trans. on Information Forensics and Security, 1(3):395-399, 2006.

[4] C. Bo, L. Zhang, and X. Li. Silentsense: Silent user identification via dynamics of touch and movement behavioral biometrics. Proc. of MobiCom, 2013.

[5] A. Chan, T. Halevi, and N. Memon. Touchpad input for continuous biometric authentication. Communications and Multimedia Security, Springer Berlin Heidelberg, pages 86-91, 2014.

[6] DataGenetics. Pin analysis. 'http://www.datagenetics.com/blog/september32012/', April 2015.

[7] D. Davis, F. Monrose, and M. Reiter. On user choice in graphical password schemes. Proc. of 13th Usenix Security Symposium, 13:1-14, 2004.

[8] R. Dhamija and A. Perrig. Deja vu: User study using images for authentication. Proc. of 9th Usenix Security Symposium, pages 14-17, 2000.

[9] A. Dirik, N. Memon, and J. Birget. Modeling user choice in the passpoints graphical password scheme. Proc. of the 3rd symposium on Usable privacy and security (SOUPS), pages 20-28, 2007.

[10] T. Feng, Z. Liu, K.-A. Kwon, W. Shi, B. Carbunar, Y. Jiang, and N. Nguyen. Continuous mobile authentication using touchscreen gestures. Proc. of IEEE Conf. Homeland Security (HST), pages 451-456, 2012.

[11] M. Frank, R. Biedert, E. Ma, I. Martinovic, and D. Son. Touchalytics: On the applicability of touchscreen input as a behavioral biometric for continuous authentication. IEEE Trans. on Information Forensics and Security, 8(1):136-148, June 2013. 
[12] T. Halevi, S. Lin, D. Ma, A. Prasad, N. Saxena, J. Voris, and T. Xiang. Sensing-enabled defenses to rfid unauthorized reading and relay attacks without changing the usage model. Proc. of PerCom, pages 227-234, 2012.

[13] T. Halevi, D. Ma, N. Saxena, and T. Xiang. Secure proximity detection for nfc devices based on ambient sensor data. Proc. of ESORICS, Lecture Notes in Computer Science, pages 379-396, 2012.

[14] H. Jo, M. Karsai, J. Kerte`sz, and K. Kaski. Circadian patterns and burstiness in mobile phone communication. New Journal of Physics, 14, 2012.

[15] F. Li, N. Clarke, M. Papadaki, and P. Dowland. Active authentication for mobile devices utilising behaviour profiling. Intl' Journal of Information Security, pages 1-16, 2013.

[16] K. Niinuma, U. Park, and A. Jain. Soft biometric traits for continuous user authentication. IEEE Trans. on Information Forensics and Security, 5(4):771-78, 2012.

[17] L. Rabiner and B. Juang. Fundamentals of speech recognition. Prentice Hall Signal Processing Series, 1993.

[18] A. Roy, T. Halevi, and N. Memon. An hmm-based behavior modeling approach for continuous mobile authentication. In Proc. of IEEE Intl' Conf. on Acoustics, Speech and Signal Processing (ICASSP), pages 3789-3793. IEEE, 2014.

[19] A. Roy, T. Halevi, and N. Memon. An hmm-based multi-sensor approach for continuous mobile authentication. In Military Communications Conference, MILCOM 2015-2015 IEEE, pages 1311-1316. IEEE, 2015.

[20] A. Roy, N. Memon, and T. Halevi. Movile active authentication survey. 'http://bit.ly/1cSjYHF', 2014.

[21] N. Sae-Bae and N. Memon. A simple and effective method for online signature verification. Proc. of Intl' Conf. of Biometrics Special Interest Group (BIOSIG), pages 1-12, 2013.

[22] N. Sae-Bae, N. Memon, and K. Isbister. Investigating multi-touch gestures as a novel biometric modality. Proc. of IEEE Fifth Intl' Conf. on Biometrics: Theory, Applications and Systems (BTAS), 14:156-161, 2012.

[23] N. Sae-Bae, N. Memon, and K.Isbister. Biometric-rich gestures: A novel approach to authentication on multi-touch devices. Proc. of ACM Annual Conf. on Human Factors in Computing Systems (CHI), pages 977-986, 2012.

[24] A. Serwadda, Z. Wang, P. Koch, S. Govindarajan, and R. P. et al. Scan-based evaluation of continuous keystroke authentication systems. IT Professional, 15(4):20-23, 2013.

[25] W. Shi, J. Yang, Y. Jiang, F. Yang, and Y. Xiong. Senguard: Passive user identification on smartphones using multiple sensors. Proc. of IEEE rth Intl' Conf. on Wireless and Mobile Computing, Networking and Communications(WiMob), pages 141-148, 2011.

[26] T. Van Nguyen, N. Sae-Bae, and N. Memon. Finger-drawn pin authentication on touch devices. In Image Processing (ICIP), 2014 IEEE International Conference on, pages 5002-5006. IEEE, 2014.
[27] T. Van Nguyen, N. Sae-Bae, and N. Memon. Draw-a-pin: Authentication using finger-drawn pin on touch devices. Computers \& Security, 66:115-128, 2017.

[28] D. Weinshall and S. Kirkpatrick. Passwords you'll never forget, but can't recall. Proc. of ACM Annual Conf. on Human Factors in Computing Systems(CHI), pages 1399-1402, 2004.

[29] R. Woo, A. Park, and T. Hazen. The mit mobile device speaker verification corpus: data collection and preliminary experiments. Proc. of IEEE 'th Intl' Conf. on The Speaker and Language Recognition Workshop, pages 1-6, 2006. 